\title{
Median Sternotomy for the Management of Life-Threatening Bleeding Resulting from Proximal Upper Extremity Amputation
}

\author{
Hyunseong Kang a, Gyu Bum Seo a, Su Wan Kim b,* \\ a Department of Orthopedics, Jeju National University Hospital, Jeju National University School of Medicine, Jeju, Korea \\ ${ }^{b}$ Department of Thoracic and Cardiovascular Surgery, Jeju National University Hospital, Jeju National University School of Medicine, Jeju, Korea
}

\section{Article history:}

Received: April 17, 2019

Accepted: August 22, 2019

\section{${ }^{*}$ Corresponding Author:}

Su Wan Kim

Department of Thoracic and Cardiovascular Surgery, Jeju National University Hospital,

Jeju National University School of Medicine, Jeju, Korea

E-mail: 95swan@hanmail.net

\section{ORCID}

Hyunseong Kang

https://orcid.org/0000-0001-6764-9929

Gyu Bum Seo

https://orcid.org/0000-0002-5854-1606

Su Wan Kim

https://orcid.org/0000-0002-6531-7266

\begin{abstract}
It is extremely difficult to achieve hemostasis in the case of proximal amputation of major limbs, and especially in the case of a proximal upper arm amputation, which is adjacent to the axilla. A 30-yearold male sustained a traumatic proximal left upper extremity amputation resulting in hypovolemic shock. A median sternotomy was performed to clamp the left subclavian artery. The completely transected brachial artery was reconstructed with an artificial vascular graft and the transected nerves were repaired. With simultaneous rehabilitation, the patient recovered from the hypovolemic shock and ischemic injury of the arm. Performing a median sternotomy for proximal clamping of the transected artery allowed control of the bleeding which could not be controlled by a pressure cuff. This procedure may be considered a safe and effective surgical option for patients with traumatic proximal upper extremity amputation.
\end{abstract}

Keywords: amputation, sternotomy, wounds and injuries

\section{Introduction}

The incidence of limb amputation is common amongst major trauma patients who are admitted to the emergency room of a hospital facility. Since limb amputation itself can cause death of the patient as a result of massive hemorrhage, immediate hemostasis is required. The hemostasis of most amputation bleeding can be achieved by applying a pressure cuff at the proximal portion of the injury site. However, an effective hemostasis method for many proximal amputation sites is challenging, especially when it is adjacent to the axillary region of the upper limb. In this report, an incomplete upper limb amputation in the axillary region due to trauma is presented where a successful median sternotomy and hemostasis was performed on a patient who sustained hypovolemic shock due to a massive hemorrhage.

\section{Case Report}

A previously healthy 30-year-old male patient was admitted to the emergency room sustaining a massive hemorrhage from the left arm, due to an injury resulting from a serious fall which occurred whilst in a drunken state. The patient collided into a glass window with his left arm outstretched at the time of the fall. The broken glass deeply penetrated into the medial aspect of the biceps from the axillary area. By the time the paramedics arrived, there was already a large volume of blood lost from the patient. The systolic blood pressure of the patient was recorded as $60 \mathrm{mmHg}$ at that time. His blood pressure could not be checked upon arrival at the emergency room. The heart rate was recorded as 133 beats/minute, and his consciousness became stuporous. During transport to the hospital, the wound was wrapped with a towel. When the towel was removed in the emergency room to confirm the wound, a large eruptive bleeding occurred. It was difficult to perform a visual in-depth confirmation of the wound 
and was therefore immediately wrapped with a compression bandage. After inserting a central venous catheter and infusion of a large volume of normal saline, the patient's blood pressure increased to $104 / 64 \mathrm{mmHg}$ and heart rate was stabilized at 112 beats/minute. At that time, concentrated red blood cells, and concentrated plasma and platelets (fresh frozen) were administered and a computed tomoangiography was performed, to observe the vascular status of the left arm. Findings showed a rupture of the connecting segment of the left axillary artery and the brachial artery, accompanied by a massive hematoma surrounding the muscles and soft tissues (Figure 1).

Consequently, an emergency operation was performed on the patient. Initially, the procedure of clamping of the axillary artery via a subclavian incision was considered. However, adequate exposure and approach was deemed unattainable, due to the excessive volume of hematoma in the pectoralis muscles, as shown in Figure 2A. Hence, a sternotomy was performed to gain a superior visual field and access to the region. The left arm was placed in abduction while the patient was in a supine position under general anesthesia. A pressure cuff could not be applied around the axillary area while the hemorrhage persisted. A median sternotomy was performed to clamp the proximal portion of the left subclavian artery. The thymus was lifted and the pericardium was opened vertically. The innominate artery, left common carotid artery, and left subclavian artery were identified as well as the vagus nerve and the recurrent laryngeal nerve. After infusing 3,000 IU of heparin intravenously and clamping the left subclavian artery using a vascular clamp, the bandage surrounding the axilla was removed to allow a more comprehensive review and visualization of the wound. A complete rupture of the biceps brachii muscle and partial rupture of the triceps brachii muscle were observed at that time. It was observed that the median nerve, radial nerve, ulnar nerve, and the brachial artery were completely severed. The ruptured ends of the brachial artery were contracted into the muscles and therefore, they could not be identified on visual review. The proximal and distal portions of the truncated brachial artery were secured by dissecting the tissue, and each was held by vascular clamps. The surgery included an end-to-end anastomosis, which was performed through multiple interrupted sutures with 7-0 polyprophylene, using an expanded polytetrafluoroethylene vascular graft (4-7 mm, Medi-Tec Excel Soft; Boston Scientific, Marlborough, MA, USA; Figure 2A). Multiple interrupted sutures with 5-0 polyprophylene were used for end-to-end anastomosis of each nerve. Additionally, the autologous basilic vein graft was used for reconstruction of the radial nerve due to a $2 \mathrm{~cm}$-sized defect. After the procedure was performed to manage the muscle repair, the skin was closed with a silicone drainage tube in place (Figure 2B).

The patient stabilized and was weaned off the ventilator within 4 hours postoperatively. He was transferred from the intensive care unit to a general ward 4 days later. The patient was prescribed $100 \mathrm{mg}$ of aspirin, $100 \mathrm{mg}$ of cilostazol, and rehabilitation treatment for limitation of movement in the arm and fingers caused by extensive nerve injuries. The blood flow in the left arm was well established.
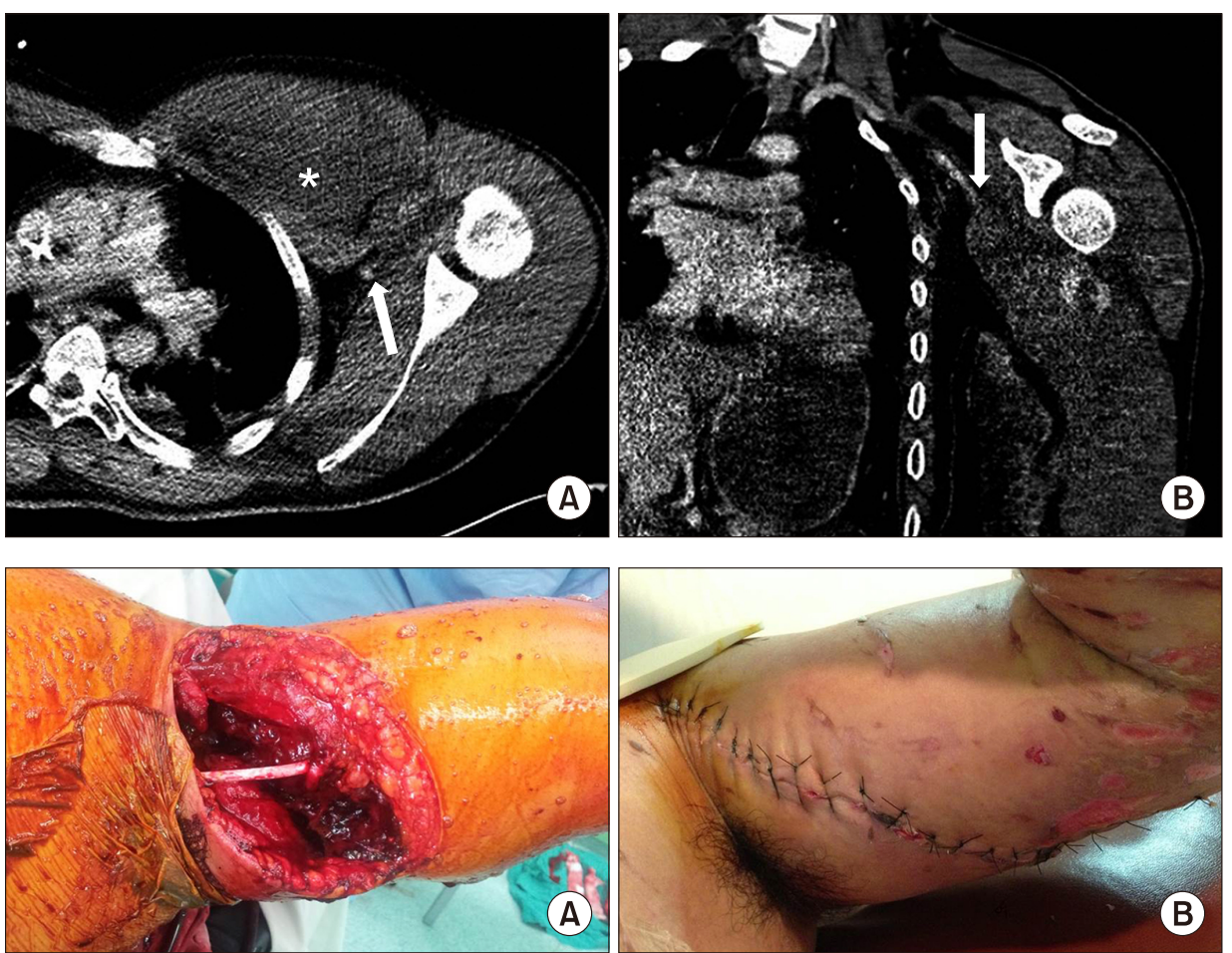

Figure 1. White arrows on Figure 1. (A) and (B) indicate the distal end of the injured brachial artery which is located at the same level with the humeral head.

*A large amount of hematoma exists in the left pectoralis muscles.

Figure 2. After a median sternotomy, the wound which was near the axilla, could be inspected. The brachial artery was completely transected and contracted into the muscles. (A) The artery was reconstructed using endto-end anastomosis with a vascular graft. (B) Seven days after the operation, the closed wound was extensive from the anterior axillary line to the elbow. 

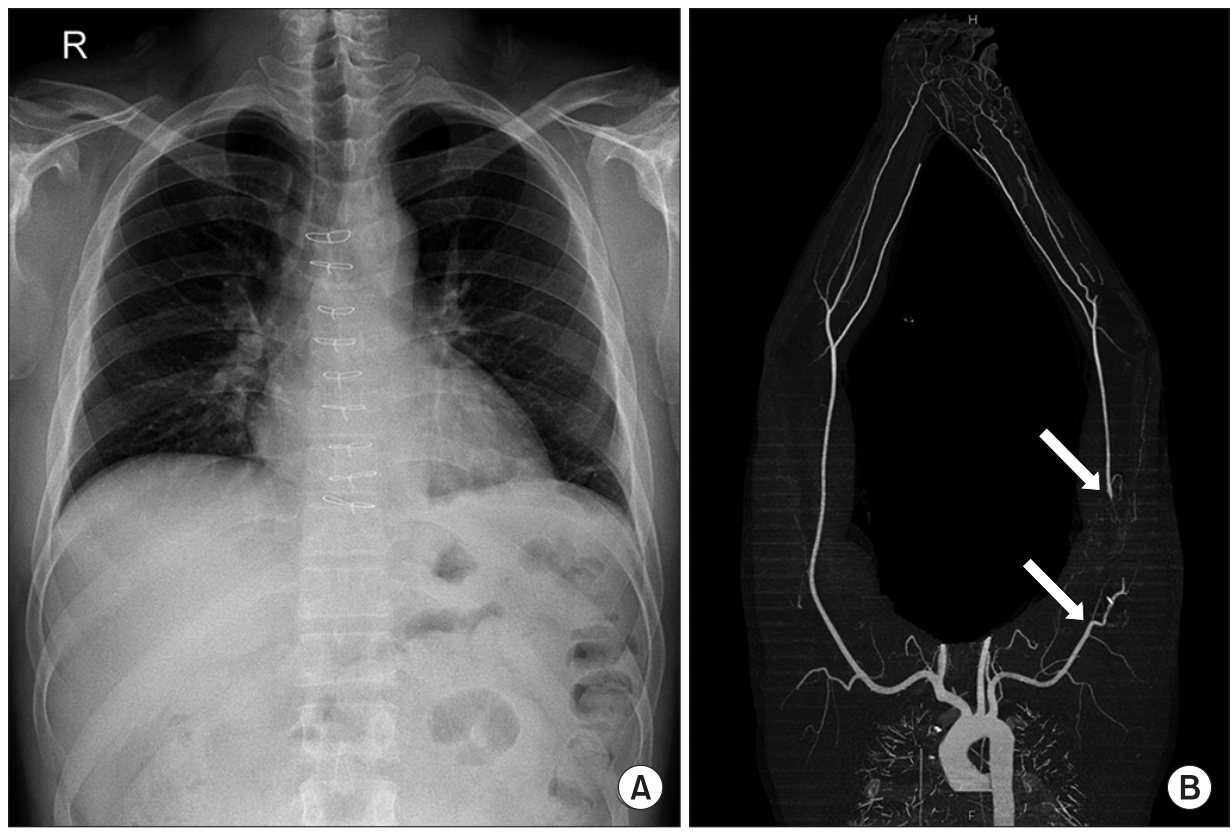

Figure. 3. (A) A chest X-ray showed no abnormal findings after the sternotomy. (B) Eighteen months after the operation, a computed tomoangiography revealed a complete obstruction of the repaired brachial artery, because of thrombosis in the vascular graft. White arrows indicate the end of the obstructed brachial artery, and the distal brachial artery is reconstructed by several collateral arteries.

The patient was discharged 87 days after the operation. There was no complication associated with the sternotomy during the follow-up period (Figure 3A). However, at the 18-month followup, a computed tomoangiography scan showed a complete obstruction of the graft in the brachial artery (Figure 3B). Several collateral vessels had been reconstructed in the distal part of the brachial artery. Although there was no necrosis or atrophy of the tissue in the arm there was limitation of movement in the left arm resulting from nerve damage that persisted at that time. The patient was recommended additional bypass surgery to the brachial artery, with regular scheduled follow-ups.

\section{Discussion}

Extensive epidemiological research on amputees is being conducted worldwide. An epidemiological study in Korea included 4,258 amputees who either had an amputation and/or received prosthetic training at a single institution from January 1970 to June 1994 [1]. Interestingly, the most common cause of amputation was trauma (66.7\%), and the second most common cause was peripheral vascular disease. While amputations due to infection or trauma were the most common in patients in the 1950s, amputations due to peripheral vascular disease have gradually increased until they now make up $23.5 \%$ of all amputations performed by surgical teams in the 1990s. Lower extremity amputation, more common than upper extremity amputation, accounted for $68.7 \%$ of all amputations. The study of Kim et al [1] showed that the various causes of amputations are subject to change according to the circumstances of the modern cultural times.
In complete and incomplete amputations alike, damage to vital structures is almost always anticipated. The most frequently observed signs of this type of damage are hemorrhage, neurological disorders, and a peripheral pulse deficit. The study of Andreev et al [2] of 50 patients with arterial injuries of the upper extremity which was reported in 1992, observed that in most cases the brachial artery was the most frequently injured. It has been reported in 2010 that traumatic limb amputation was associated with significant morbidity [3]. Although the amputation itself may not be fatal, a massive hemorrhage from associated arterial injuries should be regarded as such if not properly managed by a professional healthcare team or medical facility. The incidence of arterial injuries has shown a progressive increase during the past decade. It was reported in 1971 that among 442 cases of significant arterial injuries, 53.3\% of these were upper extremity injuries (subclavian, axillary, brachial, radial, and ulnar) [4]. Although in those identified cases, a single limb amputation did not impact patient mortality, the need for multiple limb amputation was an independent risk factor for morbidity. In that case, the mortality rate was as high as $10.4 \%$ considering all arterial injuries $[3,4]$.

To minimize any fatal hemorrhage and to reduce the mortality rate arising from traumatic amputations, exhaustive efforts for hemostasis of amputation sites are crucial until definitive repair of arterial injuries can be surgically performed on the patient. In general, the management of traumatic amputations is no different from the management routines for other trauma patients. The priority is to control major bleeding immediately $[5,6]$. However, in this current case, the amputation injury caused a junctional hemorrhage which precluded the effective use of a conventional tourniquet. 
A junctional hemorrhage is defined as a hemorrhage that occurs at the junction of an extremity with the torso of the body at an anatomic location, that may include bleeding from the groin proximal to the inguinal ligament, the buttocks, the gluteal and pelvic areas, the perineum, the axilla and shoulder girdle, and the base of the neck. In 2013 it was proposed by Kotwal et al [7]. that an extremity tourniquet for a junctional hemorrhage was ineffective at controlling the bleeding. A junctional hemorrhage is more lethal than more distal limb injuries. With preventable deaths from extremity hemorrhages greatly reduced by tourniquet use, the junctional hemorrhage is the leading cause of death from external hemorrhages. In an effort to control junctional hemorrhages, junctional tourniquets have been made available for medical use.

Although many emergency providers testify to the success of junctional tourniquets for paramedic use, this is an area of ongoing evaluation to prove that these devices improve patients' outcome [5,6]. Kim et al [8] reported in 2015 a hybrid approach for treating subclavian artery laceration caused by the removal of a pigtail pleural drainage catheter in a patient with a pneumothorax. The patient was successfully resuscitated through diagnostic angiography with subsequent balloon occlusion and primary repair of the injured subclavian artery. The controlled management of hemorrhages in the axillary area remain a challenge for many surgeons. A median sternotomy provides a more extensive visual field advantageous in accessing the vascular structures compared to other approaches. That being said, the surgeon should be cautious not to cause iatrogenic injury during the routine dissection, especially in relation to the laryngeal nerve and the vagus nerve [9]. Although the reported incidence of iatrogenic injury remains low, a median sternotomy and/ or sternal traction pulling laterally on both subclavian arteries, and direct manipulation and retraction during open-heart procedures, are known to be possible mechanisms of associated complications, such as vocal cord paralysis [10]. Additionally, a bolus administration of heparin prior to clamping of the artery would be beneficial to the patient.

In the patient in this current study, the antiplatelet effect of 100 $\mathrm{mg}$ of aspirin and $100 \mathrm{mg}$ of cilostazol proved to be insufficient to maintain the patency of the repaired brachial artery. A study in 1971 [4] showed that resection and anastomosis under tension, or stenosis at the anastomotic site was the most common cause of failure of this procedure. With the advent of a perioperative anticoagulation therapy with heparin, the incidence rate of development of wound hematomas increased, but long-term anticoagulation therapy with warfarin was reported in 1998 to improve the patency rate of the autogenous vein infra-inguinal bypass grafts, and the limb salvage rate for patients at high risk of graft failure [11]. Further research on the opportunity for perioperative therapy to maintain vascular patency is needed in these cases. It can be reported that warfarin and/or other anticoagulation regimens should be considered for use with traumatic amputation patients who present with concomitant arterial injuries.

In conclusion, we suggest that the median sternotomy for the proximal clamping of the transected artery is a safe and effective surgical option for patients with a traumatic proximal upper extremity amputation.

\section{Conflicts of Interest}

No potential conflict of interest relevant to this article was reported.

\section{Acknowledgements}

This work was supported by the 2020 education, research and student guidance grant funded by Jeju National University.

\section{References}

[1] Kim YC, Park CI, Kim DY, Kim TS, Shin JC. Statistical analysis of amputations and trends in Korea. Prosthet Orthot Int 1996;20(2):88-95.

[2] Andreev A, Kavrakov T, Karakolev J, Penkov P. Management of acute arterial trauma of the upper extremity. Eur J Vasc Surg 1992;6(6):593-8.

[3] Barmparas G, Inaba K, Teixeira PG, Dubose JJ, Criscuoli M, Talving P, et al. Epidemiology of post-traumatic limb amputation: A National Trauma Databank Analysis. Am Surg 2010;76(11):1214-22.

[4] Perry MO, Thal ER, Shires GT. Management of arterial injuries. Ann Surg 1971;173(3):403-8.

[5] Rush RM Jr, Arrington ED, Hsu JR. Management of complex extremity injuries: Tourniquets, compartment syndrome detection, fasciotomy, and amputation care. Surg Clin North Am 2012;92(4):987-1007.

[6] Kragh JF Jr, Walters TJ, Baer DG, Fox CJ, Wade CE, Salinas J, et al. Practical use of emergency tourniquets to stop bleeding in major limb trauma. J Trauma 2008;64(2 Suppl):S38-49; discussion S49-50.

[7] Kotwal RS, Butler FK, Gross KR, Kheirabadi BS, Baer DG, Dubick MA, et al. Management of junctional hemorrhage in tactical combat casualty care: TCCC guidelines? Proposed change 13-03. J Spec Oper Med 2013;13(4):8593.

[8] Kim HJ, Cho YH, Suh GY, Yang JH, Jeon K. Subclavian artery laceration caused by pigtail catheter removal in a patient with pneumothorax. Korean J Crit Care Med 2015;30(2):119-22.

[9] Hoballah JJ. Vascular reconstructions: Anatomy, exposures, and techniques. New York (NY): Springer; 2000. p. 17-26.

[10] Hamdan AL, Moukarbel RV, Farhat F, Obeid M. Vocal cord paralysis after open-heart surgery. Eur J Cardiothorac Surg 2002;21(4):671-4.

[11] Sarac TP, Huber TS, Back MR, Ozaki CK, Carlton LM, Flynn TC, et al. Warfarin improves the outcome of infrainguinal vein bypass grafting at high risk for failure. J Vasc Surg 1998;28(3):446-57. 